\title{
Endoscopic Ultrasound-Guided Random Omental Fine Needle Aspiration: A Novel Technique for the Diagnosis of Peritoneal Carcinomatosis
}

\author{
Pradeep Kumar Siddappa', Neha Jain ${ }^{1}$, Naveen K. Agarwal ${ }^{2}$, Monika Jain ${ }^{1}$ and Gurwant Singh Lamba ${ }^{1}$ \\ ${ }^{1}$ Department of Gastroenterology, Sri Balaji Action Medical Institute, New Delhi, ${ }^{2}$ Department of Pathology, Action Cancer Hospital, \\ New Delhi, India
}

Background/Aims: Diagnostic abdominal paracentesis has been described in literature to have variable sensitivity of 50\%-75\% for the detection of peritoneal carcinomatosis (PC). We believe that random needle aspirates from the omentum, even in the absence of obvious deposits by endoscopic ultrasound (EUS), could prove malignancy in patients with PC.

Methods: Consecutive patients who underwent EUS for diagnosis and staging of cancer and found to have ascites were included after obtaining informed consent. EUS-guided fine needle aspiration (EUS-FNA) from random sites in the omentum was performed through the transgastric route using a linear echoendoscope.

Results: Fifty-four patients underwent EUS during October 2015 to April 2017 for detection, staging, or FNA of a suspected malignant lesion. Ascites was seen in 17 patients and 15 patients who fulfilled the criteria were included. The procedure was successful in all patients. Cytology was suggestive of malignancy in 12 (80\%) but not suggestive of malignancy in 3 (20\%) patients. Three patients who tested negative had hyperbilirubinemia with biliary obstruction. Their ascitic fluid analysis result was also negative.

Conclusions: Random FNA of the omentum in patients with malignancy-related ascites is highly effective in the diagnosis of PC and could be employed during EUS evaluation of malignancies. Clin Endosc 2020;53:594-599

Key Words: Ascites; Endoscopic ultrasound; Endoscopic ultrasound-guided fine needle aspiration; Omentum; Peritoneal neoplasms

\section{INTRODUCTION}

Diagnostic abdominal paracentesis has been described in literature to have variable sensitivity of $50 \%-75 \%$ for the detection of peritoneal carcinomatosis (PC) in malignancy related ascites. ${ }^{1-3}$ Endoscopic ultrasound-guided fine needle aspiration (EUS-FNA) of peritoneal deposits have been shown to detect

Received: September 9, 2019 Revised: March 20, 2020

Accepted: April 3, 2020

Correspondence: Pradeep Kumar Siddappa

Current affiliation- Department of Internal Medicine, UCONN Health, 263 Farmington Avenue, Farmington, CT 06030, USA

Tel: +1-507-202-4577, Fax: +1-860-679-4613, E-mail: dr.pradeep@outlook.com

ORCID: https://orcid.org/0000-0001-9634-9028

(c) This is an Open Access article distributed under the terms of the Creative Commons Attribution Non-Commercial License (http://creativecommons.org/ licenses/by-nc/3.0) which permits unrestricted non-commercial use, distribution, and reproduction in any medium, provided the original work is properly cited.
PC. ${ }^{4}$ Detection of metastatic deposits and their aspiration requires a high level of expertise and skill to achieve consistent results. We believe that random EUS guided needle aspirates from the omentum, with or without visible peritoneal deposits on EUS can help diagnose patients with PC. In this pilot study, we aim to show the $\mathrm{PC}$ detection rate in patients undergoing EUS-FNA from random sites on the omentum with ascites and proven or suspected malignancy.

\section{MATERIALS AND METHODS}

This pilot, single arm prospective uncontrolled study, was conducted at a tertiary hospital in New Delhi from October 2015 to April 2017. The study was approved by the institutional ethics committee. Consecutive patients with ascites and known or suspected malignancy, who underwent EUS 
for staging or FNA, were included after obtaining informed consent. Patients who did not have a vessel free window on EUS for the safe introduction of FNA needle, who needed the needle to traverse the mass/tumor involving the stomach or duodenal wall to reach the omentum were excluded.

Patients were considered to have PC if they had, (1) Cytological analysis of EUS-FNA of the omentum confirming malignant cells or showing cells suspicious of malignancy in

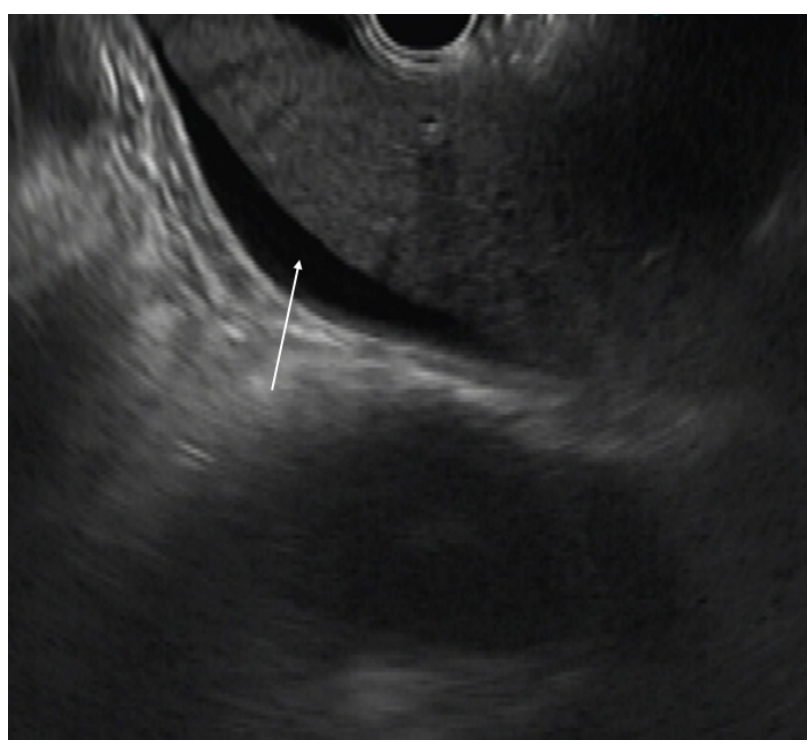

Fig. 1. Endoscopic ultrasound image showing ascites. Ascites visualized as the anechoic area around the liver (arrow).

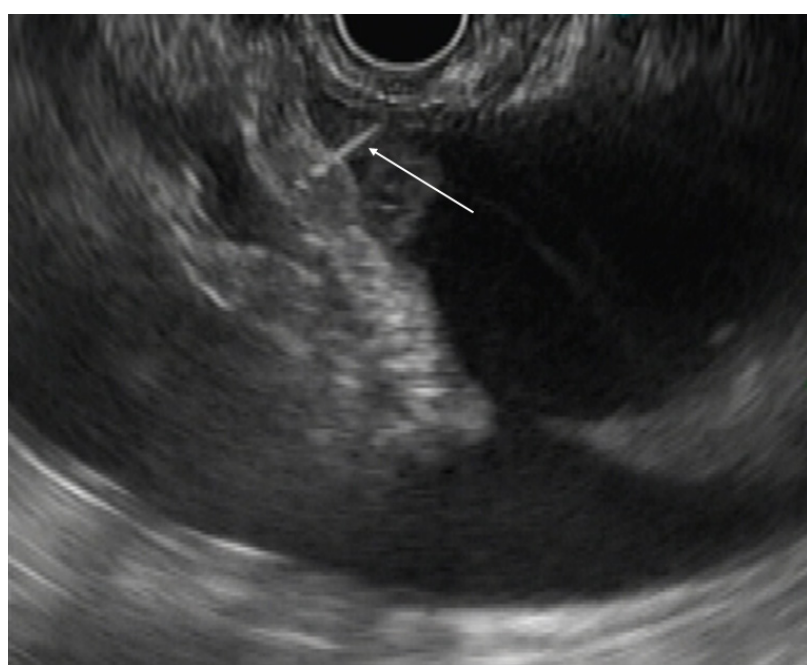

Fig. 3. Endoscopic ultrasound (EUS) image showing EUS needle penetrating the omentum. Fine needle aspiration needle penetrating the omentum (arrow). the right clinical context, (2) Ascitic fluid cytology positive for malignant cells, (3) Surgical histopathology confirming the presence of peritoneal involvement.

Consenting patients underwent EUS using a liner echoendoscope (GF-UCT180; Olympus, Tokyo, Japan). The procedure was performed under conscious sedation. Ascites has an anechoic appearance on EUS (Fig. 1). Omentum was identified as a frond-like hyperechoic, floating, intraperitoneal

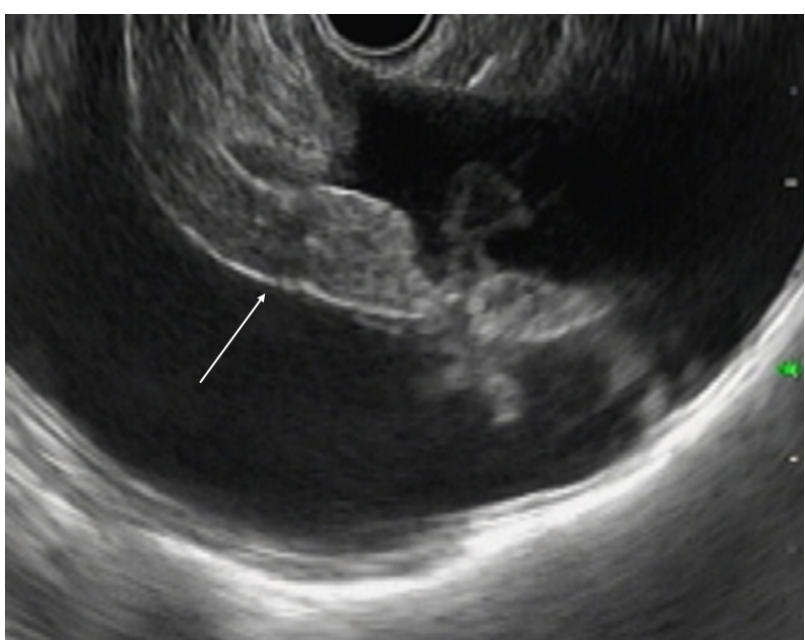

Fig. 2. Endoscopic ultrasound image showing the omentum. Frond-like omentum in the middle of ascites (anechoic, area) (arrow).

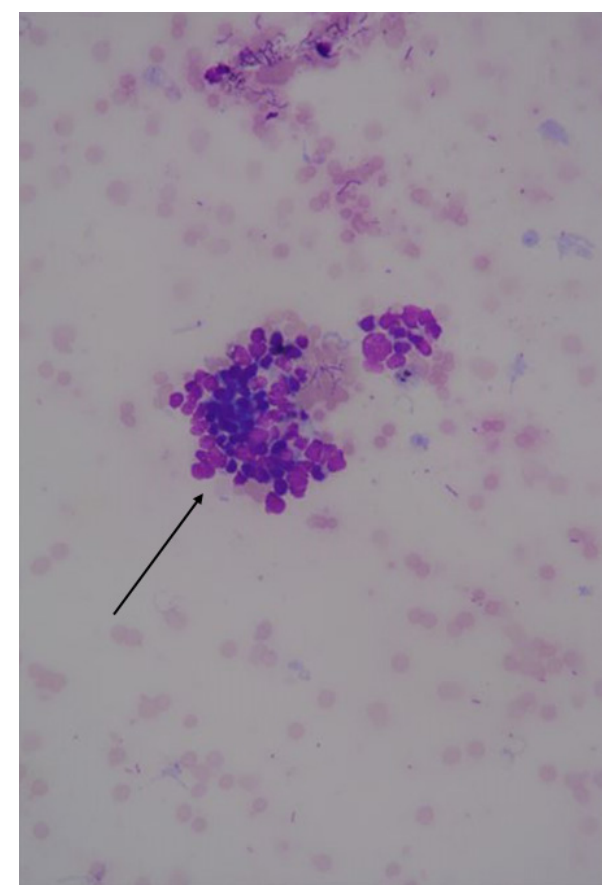

Fig. 4. Malignant cells obtained from the needle aspiration of the omentum (hematoxylin-eosin, original magnification $\times 40$ ). 
structure on EUS evaluation when viewed from the stomach station, in the background of ascites (Fig. 2). A clear and safe path, away from the liver and blood vessels in the space between the stomach wall and the omentum, with intervening ascites, was identified on EUS. EUS-FNA was performed from the omentum through the transgastric route. A 22-gauge (G) or 25 G FNA needle (Expect; Boston Scientific Co., Natick, MA, USA) was used for all the procedures. FNA was only attempted if the needle did not traverse the organ involved in the malignant process. EUS-FNA needle was carefully introduced under the vision to avoid liver and vessels within the omentum (Fig. 3). Rapid quick jabs were required to pierce through the stomach wall and reach the omentum. No aspiration was applied to the FNA needle to ensure that the aspirate was minimally contaminated by ascitic fluid. Smears were made from the material aspirated and sent for analysis (Fig. 4). Two or more passes were taken from random sites on the omentum. We did not have a cytopathologist or a cytotechnologist for assessment of adequacy at the bedside during any of the procedures.

Patients were monitored for 2 hours after the procedure in the endoscopy suite for any complication. Each patient was given a short course of post-procedure oral antibiotic for 3 days. Percutaneous trans-abdominal paracentesis was performed for patients who had negative results on EUS-FNA. Computed tomography (CT) or magnetic resonance imaging findings of the involvement of the omentum were recorded for each patient. EUS-FNA results showing the presence of malignant cells or smears suspicious for malignancy were considered diagnostic of PC in the right clinical context.

\section{Statistical analysis}

Categorical variables were expressed as frequency (\%) and continuous variable were reported as median (range).

\section{RESULTS}

Fifty-four patients underwent EUS during the study period for detection, staging or FNA of the malignant lesion. Ascites was seen in 17 patients. Two patients who refused FNA were excluded. Eventually, 15 patients who fulfilled the criteria were included (Table 1). The median age of the patients was 64 (range, 33-80) years and 11 (73.3\%) of them were females. Cross-sectional imaging was performed before the EUS in all the patients. Ascites was noted on cross-sectional imaging in all 15 patients. Peritoneal or omental involvement was noted on cross-sectional imaging in $6(40 \%)$ patients, while no peri- toneal or omental involvement was seen in $9(60 \%)$. Peritoneal deposits/nodules were noted in 2 (13.3\%), omental thickening was seen in $2(13.3 \%)$, omental deposits are seen in $1(6.6 \%)$, and omental stranding was noted in 1 patient (6.6\%) (Table 1). The indications for EUS were: the evaluation of ascites in 11 patients $(73.3 \%)$, pancreatic ductal adenocarcinoma in 1 patient (6.6\%), and evaluation of obstructive jaundice in 3 patients (20.1\%).

Omental deposits were identified on EUS in 2 patients. In the rest of patients, omentum was identified without any obvious abnormality. All the patients underwent EUS-FNA of the omentum through the transgastric route. Adequate tissue was obtained in all the patients. Patients underwent a median of 3 passes (range, 2-5) from the omentum. None of the patients underwent EUS guided trans-gastric ascitic fluid aspiration. Omental tissue appeared as translucent bits of tissue on the slide after transfer from the FNA needle.

Cytology was suggestive of malignancy in 12 patients (80\%) and not suggestive of malignancy in 3 (20\%). The final report was positive for malignancy in 10 , suspicious in 2 , and negative in 3 patients. Immunohistochemistry was performed and identified adenocarcinoma in 6 and was not performed in the rest. Primary malignancy was ovarian in 4 patients, gall bladder in 3 patients, unknown primary in 1 patient, and cholangiocarcinoma in 2 patients (Table 1).

The three patients with negative results had high-grade common bile duct obstruction with total bilirubin levels of $299.25 \mu \mathrm{mol} / \mathrm{L}, 244.5 \mu \mathrm{mol} / \mathrm{L}$, and $273.6 \mu \mathrm{mol} / \mathrm{L}$ at the time of the procedure. Ascitic fluid analysis in these 3 patients showed a serum ascites albumin gradient (SAAG) of more than $11 \mathrm{~g} /$ L. They did not have any imaging features of cirrhosis. A final diagnosis of cholangiocarcinoma was made by abdominal wall biopsy of a metastatic lesion and endoscopic retrograde cholangiopancreatography biliary biopsy (Table 1). These three patients were followed for aduration of 90, 50, and 102 days, and none of them were diagnosed with PC. We considered, both, suspicious and positive report of malignancy as being definitive evidence of malignancy. This made the sensitivity and specificity of the procedure to diagnose PC to be $100 \%$. However, if we consider patients with a suspicious report to be false negative, our procedure had a sensitivity, specificity, positive predictive value, and negative predictive value of $83.3 \%$, $100 \%, 100 \%$, and $60 \%$, respectively.

None of the patients developed any procedure-related adverse events. All the patients completed a 3-day course of antibiotics and none of them developed any signs or symptoms of infection. 


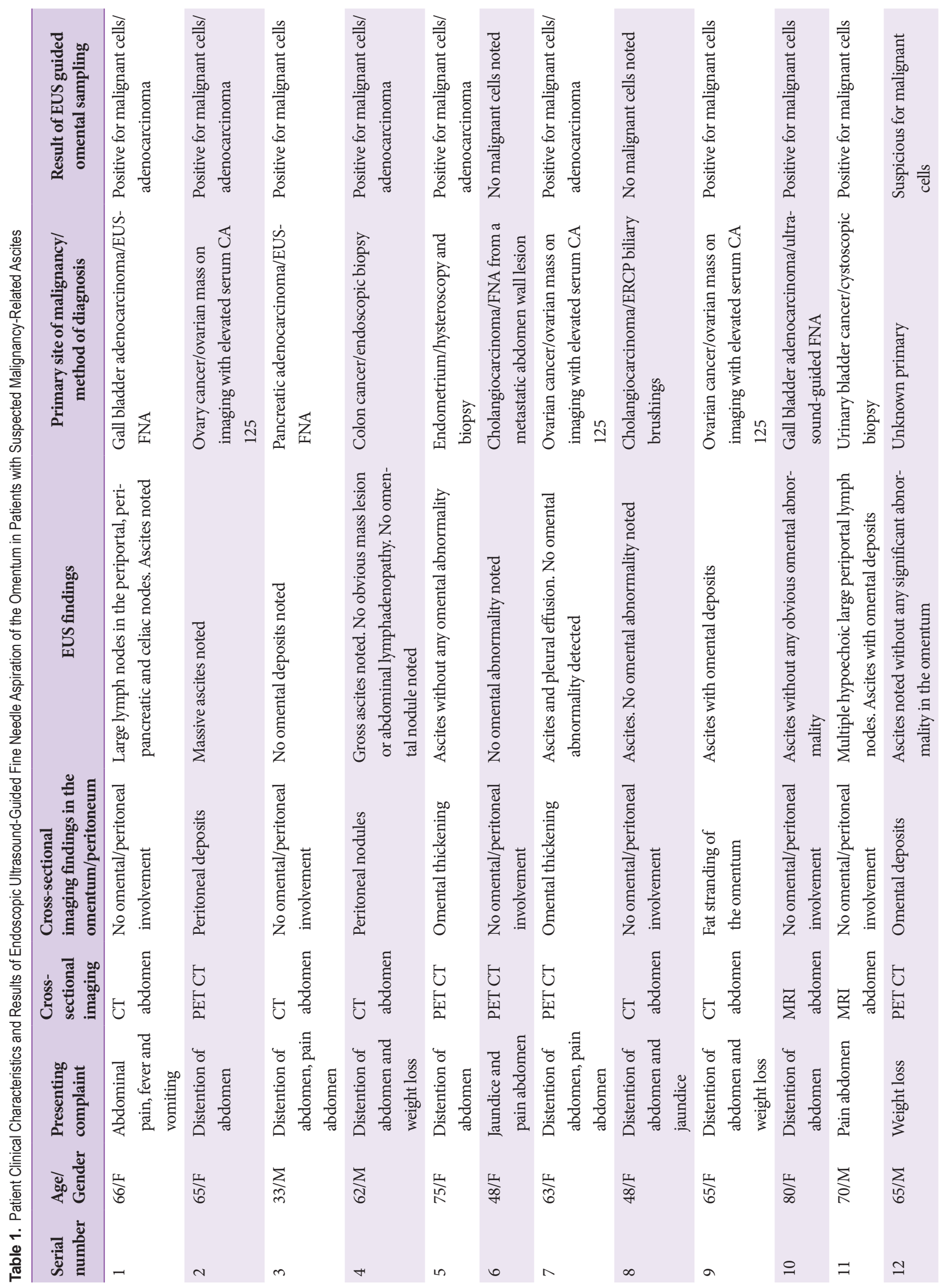




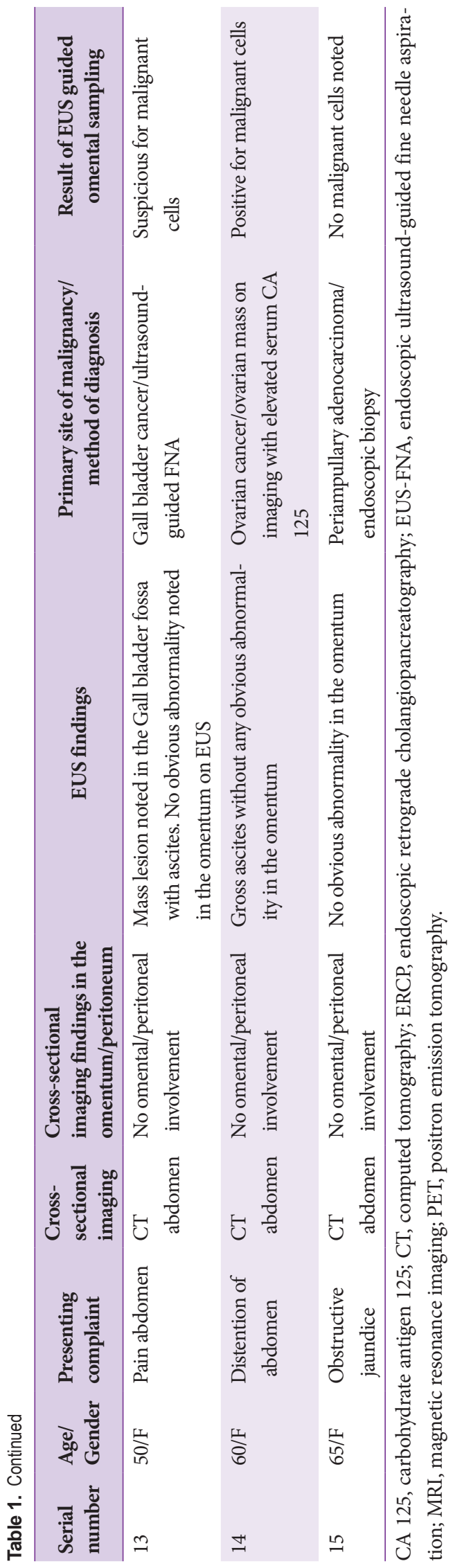

\section{DISCUSSION}

Malignancy-related ascites is a broad term for patients who develop ascites due to underlying malignancy. It accounts for less than $7 \%$ of cases with ascites. ${ }^{5}$ The underlying cause of ascites in patients with malignancy can vary. PC is one of the causes of malignancy-related ascites. The sensitivity of cytology to detect malignant cells in patients with PC in the ascitic fluid is at best $75 \%{ }^{1-3}$ Success depends on various factors like the amount of fluid aspirated, quality of the processing service, and the number of samples processed.

Ascitic fluid appears as the anechoic area between the organs. In patients with minimal ascites, fluid can be identified as an anechoic rim around the liver with patients in the left lateral position (Fig. 1). Omentum can be identified as a hyperechoic frond-like floating structure (Fig. 2). However, if the amount of ascites is low, the best position for visualization of ascitic fluid is from the antrum of the stomach. Vessels are easily identified in the omentum and care should be taken not to injure them during aspiration. As omentum is not a fixed structure, aspiration is difficult as the needle slips during the introduction. Furthermore, unlike aspiration of solid structures which are closely abutted by the stomach wall, aspiration of omentum and penetration of the stomach wall can be difficult. To overcome these problems, the needle introduction must be swift to penetrate the stomach wall. Once the needle is in the cavity, omentum can be biopsied by to and fro motion of the needle (Fig. 3). We believe that $25 \mathrm{G}$ or $22 \mathrm{G}$ needles are best suited for this procedure. Translucent tissue is identified on the slide upon successful aspiration of omentum; however, it might not be seen in all the patients.

EUS has been shown to be better than cross-sectional imaging like CT scan for the detection of ascites. In a study by Nguyen et al., $15 \%$ of the patients taken up for EUS for gastrointestinal malignancy were noted to have ascites. ${ }^{6}$ Ascites was detected in only $18 \%$ of these patients on the CT scan. ${ }^{6}$ EUS has been used in aspiration of ascitic fluid and in the sampling of peritoneal deposits for diagnosis of PC with success. ${ }^{4,7,8}$ In a prospective case series by Kaushik et al., the sensitivity and specificity of EUS aspiration of ascitic fluid for the diagnosis of PC were $94 \%$ and $100 \%$, respectively. ${ }^{7}$ In a more recent study by Wardeh et al., the sensitivity, specificity, positive predictive value, negative predictive value, and diagnostic accuracy of EUS guided paracentesis in the diagnosis of PC were $80 \%$, $100 \%, 100 \%, 95 \%$, and $96 \%$, respectively. ${ }^{9}$ Not all studies have been so optimistic. In a retrospective study from Mayo Clinic, Rochester, peritoneal anomalies detected on EUS but not the presence of ascites were suggestive of PC (odds ratio of 2.56). They also found that EUS-FNA of the peritoneum upstaged 
malignancy in $23.6 \%$ of patients. ${ }^{10}$ A study by DeWitt et al., concluded that a negative report from cytologic analysis of EUS guided ascitic fluid aspiration does not exclude the diagnosis of malignant ascites. ${ }^{11}$ EUS and EUS-FNA is a difficult technique to master. ${ }^{12}$ Detection of subtle changes noted on EUS for the visual diagnosis of peritoneal and omental deposits can be difficult even to an experienced endoscopist.

In this pilot study, we showed that EUS-FNA from omentum in patients with malignancy-related ascites can detect PC. We believe that the presence of cancer cells in the ascitic fluid circulates and deposits on the peritoneum, increasing the density of cells in the peritoneum due to constant deposition. In a retrospective surgical series, $7.1 \%$ of patients with epithelial ovarian cancer who underwent random peritoneal biopsies from normal-appearing locations during laparotomy were upstaged, and $2.7 \%$ of the patients who underwent routine omentectomy were upstaged based on microscopic metastasis over the peritoneum and omentum. ${ }^{13}$

The omental EUS-FNA procedure is safe and none of our patients developed any features of significant intra-abdominal bleeding. None of the patients experienced pain or other adverse symptoms post-procedure. Three patients, in whom FNA was negative, may have had another mechanism of ascites. All these patients had high-grade biliary obstruction with hyperbilirubinemia. It has been shown in a mouse study that high-grade biliary obstruction can lead to portal hypertension. ${ }^{14}$ This could explain the ascites and high SAAG level in these patients. The limitations of our study were the limited sample size and the fact that not all patients underwent percutaneous ascitic fluid aspiration.

To conclude, EUS guided random biopsy of the omentum in patients suspected of malignant ascites has high sensitivity and specificity for the diagnosis of PC. This procedure can be safely employed during EUS evaluation of malignancies. Further studies comparing this procedure with percutaneous ascitic fluid aspiration and surgical staging should be considered.

Conflicts of Interest

The authors have no financial conflicts of interest.

Author Contributions

Conceptualization: Pradeep Kumar Siddappa

Data curation: Neha Jain

Formal analysis: PKS

Investigation: PKS

Methodology: PKS, Naveen Kumar Agarwal
Project administration: Monika Jain, Gurwant Singh Lamba

Resources: MJ, GSL

Writing-original draft: PKS

Writing-review\&editing: NJ, NKA, MJ, GSL

ORCID

Neha Jain: https://orcid.org/0000-0002-6478-5076

Naveen Kumar Agarwal: https://orcid.org/0000-0003-3535-4311

Monika Jain: https://orcid.org/0000-0002-1103-6821

Gurwant Singh Lamba: https://orcid.org/0000-0001-8672-9537

\section{REFERENCES}

1. Allen VA, Takashima Y, Nayak S, Manahan KJ, Geisler JP. Assessment of false-negative ascites cytology in epithelial ovarian carcinoma: a study of 313 patients. Am J Clin Oncol 2017;40:175-177.

2. Decker D, Stratmann H, Springer W, Schwering H, Varnai N, Bollmann R. Benign and malignant cells in effusions: diagnostic value of image DNA cytometry in comparison to cytological analysis. Pathol Res Pract 1998;194:791-795.

3. Kielhorn E, Schofield K, Rimm DL. Use of magnetic enrichment for detection of carcinoma cells in fluid specimens. Cancer 2002;94:205-211.

4. Rana SS, Bhasin DK, Srinivasan R, Singh K. Endoscopic ultrasound-guided fine needle aspiration of peritoneal nodules in patients with ascites of unknown cause. Endoscopy 2011;43:1010-1013.

5. Runyon BA, Montano AA, Akriviadis EA, Antillon MR, Irving MA, McHutchison JG. The serum-ascites albumin gradient is superior to the exudate-transudate concept in the differential diagnosis of ascites. Ann Intern Med 1992;117:215-220.

6. Nguyen PT, Chang KJ. EUS in the detection of ascites and EUS-guided paracentesis. Gastrointest Endosc 2001;54:336-339.

7. Kaushik N, Khalid A, Brody D, McGrath K. EUS-guided paracentesis for the diagnosis of malignant ascites. Gastrointest Endosc 2006;64:908913.

8. Peter S, Eltoum I, Eloubeidi MA. EUS-guided FNA of peritoneal carcinomatosis in patients with unknown primary malignancy. Gastrointest Endosc 2009;70:1266-1270.

9. Wardeh R, Lee JG, Gu M. Endoscopic ultrasound-guided paracentesis of ascitic fluid: a morphologic study with ultrasonographic correlation. Cancer Cytopathol 2011;119:27-36.

10. Levy MJ, Abu Dayyeh BK, Fujii LL, et al. Detection of peritoneal carcinomatosis by EUS fine-needle aspiration: impact on staging and resectability (with videos). Gastrointest Endosc 2015;81:1215-1224.

11. DeWitt J, LeBlanc J, McHenry L, McGreevy K, Sherman S. Endoscopic ultrasound-guided fine-needle aspiration of ascites. Clin Gastroenterol Hepatol 2007;5:609-615.

12. Wani S, Coté GA, Keswani R, et al. Learning curves for EUS by using cumulative sum analysis: implications for American Society for Gastrointestinal Endoscopy recommendations for training. Gastrointest Endosc 2013;77:558-565.

13. Lee JY, Kim HS, Chung HH, Kim JW, Park NH, Song YS. The role of omentectomy and random peritoneal biopsies as part of comprehensive surgical staging in apparent early-stage epithelial ovarian cancer. Ann Surg Oncol 2014;21:2762-2766.

14. Franco D, Gigou M, Szekely AM, Bismuth H. Portal hypertension after bile duct obstruction: effect of bile diversion on portal pressure in the rat. Arch Surg 1979;114:1064-1067. 